\title{
Application of piezosurgery osteotomy in cervical laminoplasty: pro- spective, randomized, single-blind, clinical comparison study
}

\author{
Yanqiang Huan ${ }^{a}$, Jianfeng Liu ${ }^{\mathrm{a}}$, Wei Xing ${ }^{\mathrm{a}}$, Zhibo Zhu', Xiaolei Wang ${ }^{\mathrm{a}}$, Yongxiong $\mathrm{He}^{\mathrm{a},{ }^{*}}$
}

${ }^{a}$ Department of spinal surgery, Inner Mongolia people's hospital, Hohhot 010017, China.

\begin{abstract}
Objective: To compare and analyze the clinical efficacy of piezosurgery osteotomy and high-speed drilling respectively in cervical laminoplasty, so as to explore the operability, safety and clinical application value of piezosurgery osteotomy in cervical laminoplasty.

Methods: A total of 60 patients with multilevel cervical stenosis from the Inner Mongolia Autonomous Region People's Hospital were randomly divided into two groups (piezosurgery osteotomy group and high-speed drill group) with 30 patients in each group. Operation time, intraoperative blood loss, postoperative drainage, complication rate, Japanese orthopaedic association (JOA) score and JOA improvement rate were compared and analyzed.

Results: The operation time in piezosurgery osteotomy group and high-speed drill group was ( $87 \pm 22 \mathrm{~min})$ and (96 $\pm 35 \mathrm{~min})$, respectively, and the difference between two groups was not statistically significant $(\mathrm{P}>0.05)$. The intraoperative blood loss in the piezosurgery osteotomy group was $(121 \pm 86 \mathrm{~mL})$ lower than that (172 $\pm 93 \mathrm{~mL}$ ) in the high-speed drill group ( $<<0.05)$. The postoperative drainage in the piezosurgery osteotomy group was $(155 \pm 87 \mathrm{~mL})$ lower than that $(246 \pm 95 \mathrm{~mL})$ in the high-speed drill group $(\mathrm{P}<0.05)$. Postoperative JOA scores in both groups were better than those before surgery, but there were no significant differences in the postoperative JOA scores, improvement rates, and excellent rates between the two groups $(\mathrm{P}>0.05)$. Dural injury developed 1 patient in high-speed drill group and cerebrospinal fluid leakage occurred after surgery. No serious complications such as spinal cord injury occurred in either group.

Conclusion: Piezosurgery osteotomy in cervical laminoplasty is safe and effective, and is superior in operation time, intraoperative blood, and postoperative drainage. Its safety and efficacy are similar to high-speed drill in cervical laminoplasty.

Keywords: Cervical laminoplasty; piezosurgery osteotomy; high-speed drill; cervical spondylotic myelopathy; safety; efficacy
\end{abstract}

\section{INTRODUCTION}

Cervical spondylotic myelopathy (CSM) is the most major etiology of cervical spinal cord dysfunction. It is a cervical spondylosis with clinical dysfunction of the spinal cord based on the intervertebral disc degeneration, and usually caused by degeneration of spinal vertebral connection structures such as ossification of the posterior

*Corresponding author: Yongxiong He

Mailing address: Department of spinal surgery, Inner Mongolia people's hospital, Hohhot 010017, China.

E-mail: spinedoctor@sina.com

Received: 23 February 2020 Accepted: 27 May 2020 longitudinal ligament (OPLL), which induces the compression of the spinal cord or the spinal pathway ${ }^{[1,2]}$. It is estimated that the incidence of CSM-related hospitalizations is 4.04 per 100,000 person-years, and the number of patients undergoing surgical treatment increases by 7 times each year ${ }^{[3]}$. With the further aggravation of population aging and more and more people bending over theirs desk working for a long time, multilevel cervical spondylotic myelopathy occurring in elderly patients is no longer rare and tends to be younger. Early surgical intervention is recommended to relieve spinal cord compression, prevent aggravation of spinal cord injury, restore the function of spinal cord, and provide favorable conditions for the recovery of spinal cord function ${ }^{[4]}$. Posterior cervical expansive laminoplasty uses the 
"bowstring principle," in which the spinal cord moves in a direction with less tension, avoiding the compression in front and achieving decompression ${ }^{[5]}$. In traditional treatments, bone rongeur and high-speed drill are used for decompression, but it is difficult to control the bone drill. In addition, the cervical anatomical is complicated and congenital mutation is common, which bring great difficulties to the operation. Furthermore, these posterior decompression techniques are associated with improvements in the nervous system and visible complications, which pose a great challenge for surgeons ${ }^{[6]}$. Piezosurgery osteotomy is a new type of osteotomy technique, which has advantages of favorable tissue selectivity, osteotomy accuracy, good hemostasis, cold cutting, and easy operation. It has been widely used in the surgical field ${ }^{[7,8,9]}$.

In terms of posterior cervical expansive laminoplasty alone, whether the safety and effectiveness of piezosurgery osteotomy is superior to traditional high-speed drills has also appeared in related clinical reports ${ }^{[10]}$. However, most are retrospective studies and there is less evidence of high-quality evidence-based medicine. In this study, a 3-year randomized controlled study was conducted to compare the long-term clinical effects of cervical laminoplasty with piezosurgery osteotomy and high-speed drill, in order to provide evidence-based medical evidence to for the long-term clinical safety and efficacy of these two treatments.

\section{MATERIALS AND METHODS}

\section{Patients}

After examined and approved by the hospital ethics committee and signed the informed consent by the patient .A total of 60 patients with multilevel cervical stenosis who met the criteria for case selection were included in this study, and they were randomly divided into two groups (piezosurgery osteotomy group and high-speed drill group) with 30 patients in each group. Posterior cervical expansive laminoplasty with a titanium plate (C3-7) was performed on the surgical segments in both groups.

Inclusion criteria: (1) the diagnosis is multilevel cervical spondylotic myelopathy (Figure 1); (2) Conservative treatment is ineffective or not suitable for non-surgical treatment; (3) Two patients were operated segments perform C3-7.

Exclusion criteria: (1) secondary cervical spine surgery; (2) Cervical instability; (3) Cervical Kyphosis $\geq 10^{\circ}$; (4) Osteoporosis; (5) Cervical spine tumor, tuberculosis, infection; (6) Combined with other serious systemic diseases.

Surgical method
A

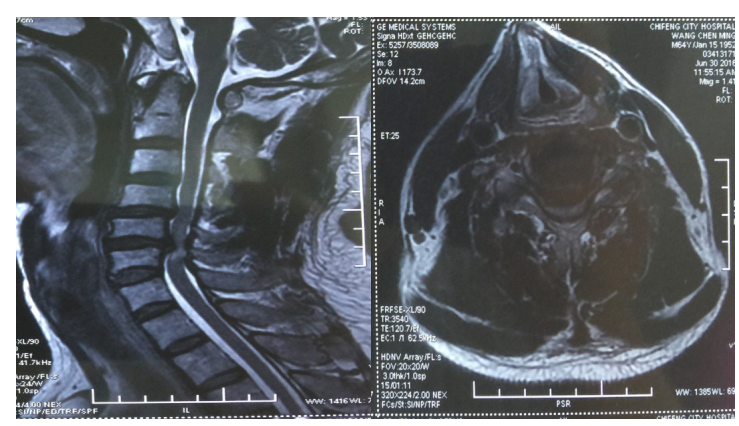

Figure 1. The cervical magnetic resonance imaging (MRI) of an illustrative case. (A)MRI showed cervical spinal stenosis and spinal cord compression degeneration. (B) MRI showed cervical spinal stenosis and severely compressed spinal cord.

General anesthesia was performed, the neck in the prone position was flexed and the head was fixed to the Mafield head frame. Through the longitudinal incision in the posterior center, the skin, subcutaneous tissue, and deep fascia was cut layer by layer to reach the spinous process along the "white line". The paraspinal muscles on both sides are peeled off under the periosteum to fully expose the lamina. The left side is the hinge side, and the right side is the opening side. According to the preoperative lottery results, the appropriate grinding head of piezosurgery osteotomy (piezosurgery osteotomy group) or spherical drill (high-speed drill group) were selected. Slots were made on the lamina of the inner edge of the left articular process, and the outer cortex of the lamina and part of the cancellous bone were ground away, exposing the inner cortical bone as a hinge (Figure 2). The vertebral lamina was grinded to the inner cortex by using grinding head of piezosurgery osteotomy and spherical drill on the medial border of right articular process (Figure 3). Replace with a spatula blade (piezosurgery osteotomy group) or Kerrison rongeur (high-speed drill

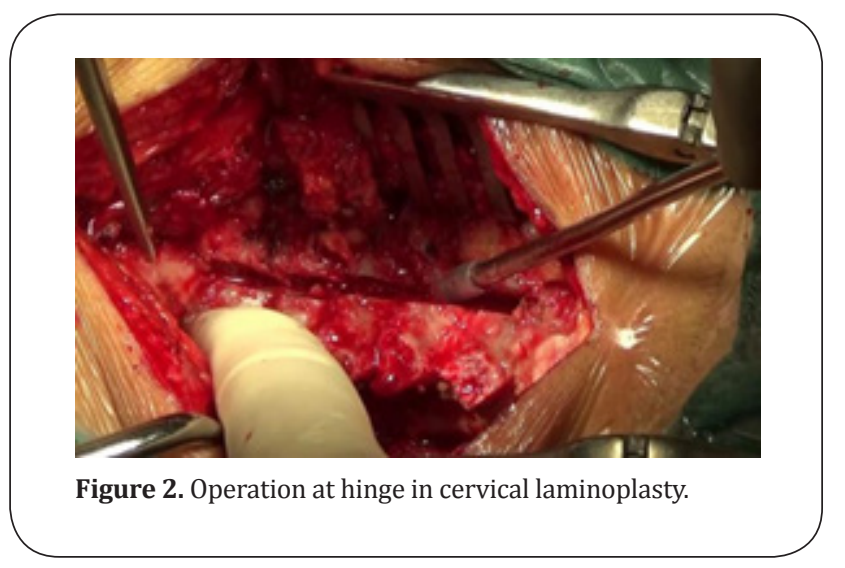




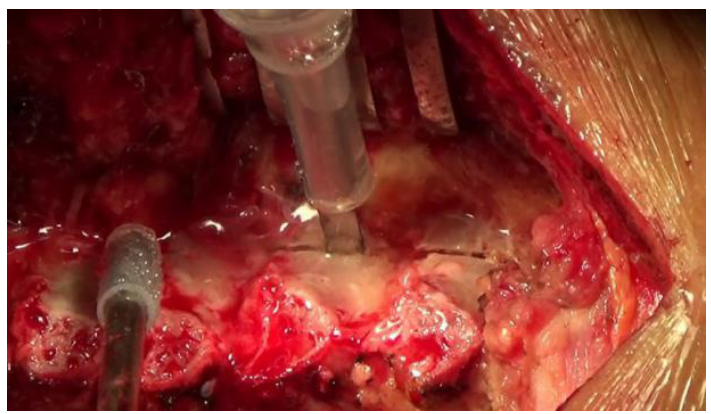

Figure 3. Operation at opening in cervical laminoplasty.

group), remove the remaining bone in the lamina slot and pry the vertebral lamina one by one, and carefully separate sticky tissue between the dural sac and the lamina. Finally, lift the lamina about $2 \mathrm{~cm}$ from the opening side to the hinge side to enlarge the spinal canal and relieve the compression of the spinal cord. Centerpiece micro-plate with appropriate length was selected to be fixed on the open side lamina and the side mass to maintain lamina openning (Figure 4). The removed spinous process cancellous bone was trimmed into broken bone particles and planted at the hinge. Clean, close the incision and place the drainage tube. After surgery, 20\% mannitol, methylprednisolone and neurotrophic drugs were routinely given for 3 days. After 3 days, patients can get out of bed under the protection of the cervical brace (Figure 5).

\section{Therapeutic evaluation}

The operation time, intraoperative blood loss, postop-

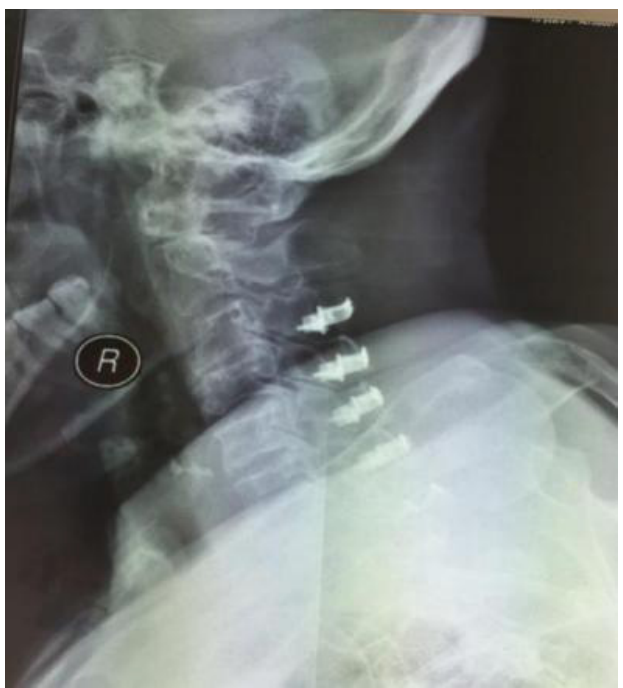

Figure 4. Centerpiece cervical laminoplasty.

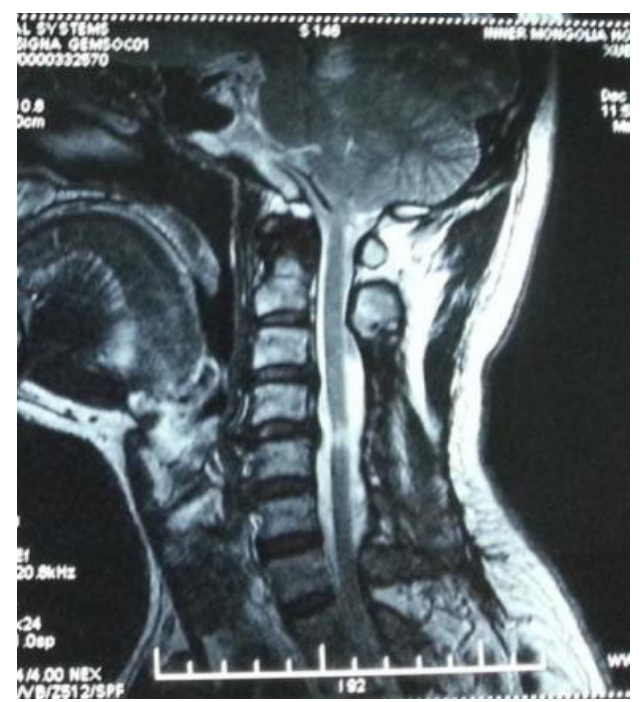

Figure 5. The magnetic resonance imaging (MRI) showed excellent postoperative swelling of the spinal cord.

erative drainage volume, and complication rate were recorded. Japanese orthopaedic association (JOA) scoring system was used to evaluate the neurological recovery of patients ${ }^{[11]}$. The JOA improvement rate was calculated based on the JOA score of the patient before and 7 days after the operation to determine the surgical effect. Excellent: improvement rate $\geq 75 \%$; good: improvement rate was from $50 \%$ to $74 \%$; medium: improvement rate was from $25 \%$ to $49 \%$; poor: improvement rate $<25 \%$.

\section{Statistical analysis}

SPSS 22.0 statistical software was used for analysis. Measurement data conforming to the normal distribution are expressed as mean \pm standard deviation ( $\overline{\mathrm{X}} \pm \mathrm{S}$ ). Two groups were compared using two independent samples t-test or paired t-test. Fisher's exact probability method was used to compare the counting data. $\mathrm{P}<0.05$ was considered statistically significant.

\section{RESULTS}

\section{Comparison of general preoperative data}

There were no statistically significant differences in the gender ( $\mathrm{p}=0.706)$, age ( 61 vs $59, \mathrm{p}=0.689$ ), and JOA score (10.0 vs $11.0, \mathrm{p}=0.184$ ) among patients who underwent the two surgical treaments (Table 1).

\section{Comparison of postoperative outcomes}

Table 2 compares clinical outcomes between patients 
Table 1. Comparison of general preoperative data between two groups of patients

\begin{tabular}{|c|c|c|c|c|c|}
\hline \multirow{2}{*}{ Group } & \multirow{2}{*}{$\begin{array}{l}\text { Case } \\
\text { number }\end{array}$} & \multicolumn{2}{|c|}{ Gender } & \multirow{2}{*}{ - Age } & \multirow{2}{*}{$\begin{array}{l}\text { JOA } \\
\text { score }\end{array}$} \\
\hline & & Male & Female & & \\
\hline $\begin{array}{l}\text { Piezosurgery } \\
\text { osteotomy group }\end{array}$ & 30 & 18 & 12 & $61 \pm 9$ & $10.0 \pm 1.5$ \\
\hline High-speed drill group & 30 & 16 & 14 & $59 \pm 11$ & $11.0 \pm 2.4$ \\
\hline Test statistic & & & & $t=0.423$ & $\mathrm{t}=1.382$ \\
\hline $\mathrm{p}$ value & & 0.706 & & 0.689 & 0.184 \\
\hline
\end{tabular}

JOA, Japanese orthopaedic association.

who underwent piezosurgery osteotomy and those who underwent high-speed drill. There were no significant difference between the two groups for operative time (piezosurgery osteotomy 87 vs high-speed drill 96, $\mathrm{p}=0.107$ ) and intraoperative blood loss (piezosurgery osteotomy 121 vs high-speed drill 172, $\mathrm{p}=0.542$ ). Postoperative drainage flow in piezosurgery osteotomy group was lower than that in high-speed drill group $(\mathrm{p}<0.05)$. The neurological function in piezosurgery osteotomy group and high-speed drill group was significantly improved, and the JOA score 7 days after the operation was significantly better than that before the operation $(\mathrm{t}=9.6727,9.571, \mathrm{P}=$ $0.000,0.000)$. However, there were no significant differences in postoperative JOA score, JOA improvement rate, and JOA excellent rate between the two groups $(\mathrm{P}>0.05)$.

\section{Complications and treatment}

Dural injury developed 1 patient in high-speed drill group, and duraplasty was performed. Cerebrospinal fluid leakage occurred after surgery, and the postoperative indwelling time of drainage tube was prolonged. We found no dural injury in piezosurgery osteotomy group, and no serious complications such as spinal cord injury occurred in either group.

\section{DISCUSSION}

In recent years, with the gradual maturity of the titani- um mini-plate technology reported by Brien ${ }^{[12]}$ for the first time and the improvement of surgical skills, posterior cervical expansive laminoplasty for the treatment of multilevel cervical spondylotic myelopathy has been widely used and achieved favorable clinical effect. Due to the low tolerance of the cervical spinal cord to minor injuries, coupled with the older age of patients with CSM who are prone to complicated those multiple basic diseases. There are mutiple and sophisticated complications in the perioperative period, and these factors have high requirements for cervical decompression operation. Traditional treatment equipment have large mechanical vibrations, long operation time, more intraoperative hemorrhage and labor intensity. It is easy to damage the dura mater and cause cerebrospinal fluid leakage after surgery. In severe cases, it can damage the spinal cord, increase the risk of surgery, and cause patients to recover slowly after surgery or even have severe sequelae. By contrast, high-speed drills have a strong cutting force, especially when cutting large bones with higher efficiency, which can quickly and precisely remove bone tissue, significantly reduce the labor intensity of the operator, improve surgical efficiency and reduce the risk of spinal cord injury ${ }^{[13]}$. However, high-speed drills also have defects such as local high temperature, excessive bone debris, and easy rolling of the surrounding soft tissue. When the drill bit rotates at a high speed, the reaction force to the handle is relatively large. In addition to the influence of the carbonized bone mud in the groove, the drill bit is prone to slippage. The operation is often interrupted by cooling operations such as irrigation and imbibition, which requires high operator technical skills ${ }^{[10]}$. In our study, there was 1 patient with dura injury in the high-speed drill group. The reasons may be high temperature and curving, combined with severe spinal stenosis and obvious dura ossification adhesion, which also indicates that the risk of high-speed drill is sometimes inevitable and new bone treatment tools are urgently needed.

As a new efficient treatment tool, the piezosurgery osteotomy plays a role in bone cutting through mechanical

Table 2. Comparison of postoperative outcomes between patients who underwent piezosurgery osteotomy versus high-speed drill

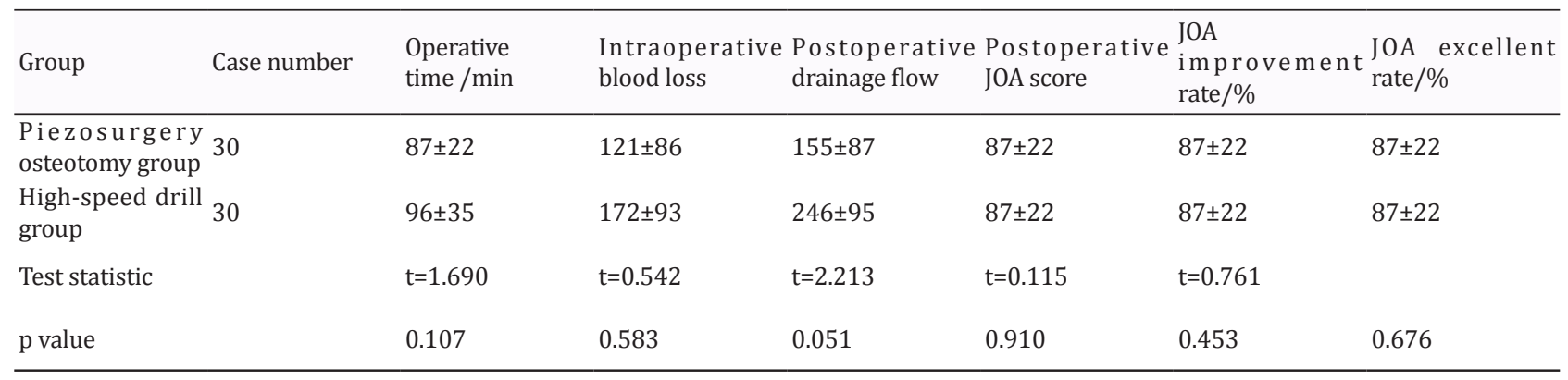

JOA, Japanese orthopaedic association. 
fragmentation effect and cavitation effect, which has the advantages of its good hemostasis, tissue selectivity, small neurovascular damage and easy operation. It was first used in oral bone treatment, and has been widely used in stomatology, plastic surgery, facial surgery, neurosurgery and orthopedics ${ }^{[7,8]}$. Hidaka first applied piezosurgery osteotomy to spinal surgery in 1998, which can reduce the incidence of nerve and dura mater injury during double-door cervical expansive laminoplasty ${ }^{[14]}$. Matsuoka et al ${ }^{[15]}$ analyzed 33 patients who underwent unilateral laminoplasty, suggesting that the ultrasonic bone knife is effective and safe for laminoplasty, and has less damage to the posterior structures such as the interspinous ligament and the supraspinous ligament. It demonstrated a postoperative spinal stability, reduced the intractable back pain caused by spinal instability and improved the postoperative satisfaction rate. Parker et al ${ }^{[16]}$ compared the traditional drill and piezosurgery osteotomy for laminoplasty. The incidence of dura injury in the former was $4.3 \%$, while that in the latter was $2.5 \%$. The results showed that piezosurgery osteotomy could reduce the risk of iatrogenic injury compared with traditional tools.

In terms of safety, the most serious complication of cervical laminoplasty is spinal cord injury. $\mathrm{Hu}^{[17]}$ found that the piezosurgery osteotomy was safe and effective in the field of spinal surgery. Some studies have shown that compared with the traditional operation, the piezosurgery osteotomy can significantly shorten the operation time ${ }^{[18]}$. Our results displayed that the operation time and intraoperative blood loss in the piezosurgery osteotomy group were significantly shorter than those in the traditional drill group $(\mathrm{P}<0.05)$. We believed that this is related to the excellent efficiency of piezosurgery osteotomy in bone cutting and its characteristic of hemostasis of surrounding bone tissue during bone cutting. High-speed drill has accessory injury to soft tissue, and the cut bone surface has no hemostatic effect. The operator should be careful to hold to prevent skidding. In this study, no spinal cord injury occurred in any of the 30 patients in the piezosurgery osteotomy group, which on the one hand indicates that the design of the surgical method assisted by piezosurgery osteotomy for cervical laminoplasty is safe, and on the other hand indicates that the application of piezosurgery osteotomy for laminectomy and decompression is safe for the spinal cord. The most common complications of decompression surgery for CSM are dural injury and cerebrospinal fluid leakage, the main cause of which is dura ossification or the dense adhesion between the dura and the ossified mass, as well as intraoperative instrument injury ${ }^{[18]}$. Studies on the use of piezosurgery osteotomy in other spinal surgeries have found that piezosurgery osteotomy can reduce the incidence of dura injuries compared with high-speed drilling, but no statistical difference has been found [19, ${ }^{20]}$. In this study, there was no dura mater injury or cerebrospinal fluid leakage due to instrument damage in the piezosurgery osteotomy group. The reason is considered that the piezosurgery osteotomy has the following two significant advantages: high tissue selectivity, no cutting effect on dura; the bone cutting mode is vibration mode, without scraping the surrounding tissue. 1 patient in the high-speed drill group suffered from cerebrospinal fluid leakage due to severe spinal canal stenosis and dural ossification. At this time, no matter whether the highspeed drill or piezosurgery osteotomy was used, dura mater injury could not be avoided. At the same time, we should also pay attention to the influence of the curve effect of the piezosurgery osteotomy learning. For those who have performed a large number of operations with high-speed drills, the application of piezosurgery osteotomy will be more handy and the learning curve will be more stable.

In terms of work efficiency, from previous studies, we can see that the piezosurgery osteotomy can shorten the operation time ${ }^{[21]}$. Nakase et al ${ }^{[22]}$ pointed out that piezosurgery osteotomy was less efficient in cutting bones than electric drills, but piezosurgery osteotomy was more efficient in cutting bones when delicate operation was carried out close to important tissue structures. Sanborn et al ${ }^{[18]}$ compared the surgical effects of piezosurgery osteotomy and high-speed drill in animal experiments of laminectomy, and piezosurgery osteotomy has advantages in terms of operation time and intraoperative blood loss. Our results indicate that the piezosurgery osteotomy can significantly shorten the average operation time of the hinged side and the open side, and better reflected its efficiency advantage when used for more segment operations. Previous studies have shown that in terms of blood loss, cancellous bone tends to continuously bleed when bone cutting is performed with a high-speed drill, and piezosurgery osteotomy is synchronized with hemostasis ${ }^{[23]}$. The bleeding tendency of the bone section was significantly reduced, which was more advantageous in intraoperative blood loss and postoperative drainage. It also indirectly reduces the occurrence of perioperative complications in a sense, and provides a guarantee for the rapid recovery of postoperative patients.

In the operation of cervical laminoplasty with piezosurgery osteotomy, the technique of the surgeon has higher requirements. In the "forming process", first of all, you need to be familiar with the anatomical characteristics of the cervical spine and the pathological characteristics of CSM, and also to be able to skillfully and accurately determine the position of the hinge side and the open side. Secondly, during the grooving process, both hands need to hold the piezosurgery osteotomy, and both hands cooperate to control the cutting depth and advance layer 
by layer. Press gently with one hand, and antagonize gently with the other to prevent cutting too deep. Hold the piezosurgery osteotomy with both hands on the opening side ${ }^{[24]}$. The operation was carried out from the spinal canal to the outside at the lateral mass joint using the ladle head to minimize intraoperative stimulation of the spinal cord. When severe spinal canal stenosis causes nerve root or dura mating between tissues to make buffer space disappear, attention should be paid to the control of cutting depth to avoid direct damage of piezosurgery osteotomy to the dura and nerve roots. Nakase et al recommend the use of electric drills in combination with piezosurgery osteotomy. High-speed drills are used for slotting the outer cortex and cancellous bone of the lamina, and it may be safer to cut the inner cortex with a piezosurgery osteotomy in a relatively large area provided by the grinding drill. Our experience is that with the use of various spoon-shaped blades, you can first use a relatively wide spoon-shaped blade to make a groove, which can also provide a relatively large area, and can predict whether the position of the groove and the door is appropriate and accurate. Incision of the inner cortex with a relatively narrow spoon-shaped knife head minimizes interference with the spinal cord and nerve roots. However, the number of cases in this study is small, and the operation time of the piezosurgery osteotomy is not recorded in real time during the operation. Prospective studies of large samples are needed in the future to further confirm the value of piezosurgery osteotomy in spine surgery.

\section{CONCLUSION}

This study has initially confirmed that the use of piezosurgery osteotomy can safely and effectively complete the operation of cervical laminoplasty, and has the advantages of less intraoperative blood, less postoperative drainage and relatively high efficiency.

\section{REFERENCES}

1. Singh, A., Tetreault, L., Casey, A., Laing, R., Statham, P., \& Fehlings, M. G. (2015). A summary of assessment tools for patients suffering from cervical spondylotic myelopathy: a systematic review on validity, reliability and responsiveness. European Spine Journal, 24(2), 209228.

2. Kim, C. H., Chung, C. K., Choi, Y., Kuo, C. C., Lee, U., Yang, S. H., ... \& Yoon, J. H. (2020). The efficacy of ultrasonic bone scalpel for unilateral cervical open-door laminoplasty: a randomized controlled trial. Neurosurgery, 86(6), 825834.

3. Bakhsheshian, J., Mehta, V. A., \& Liu, J. C. (2017). Current diagnosis and management of cervical spondylotic myelopathy. Global spine journal, 7(6), 572-586.

4. Melancia, J. L., Francisco, A. F., \& Antunes, J. L. (2014). Spinal stenosis. In Handbook of clinical neurology (Vol. 119, pp. 541-549). Elsevier.

5. Blizzard, D. J., Caputo, A. M., Sheets, C. Z., Klement, M. R., Michael, K. W., Isaacs, R. E., \& Brown, C. R. (2017). Laminoplasty versus laminectomy with fusion for the treatment of spondylotic cervical myelopathy: short-term follow-up. European Spine Journal, 26(1), 85-93.

6. Yamazaki, M., Okawa, A., Fujiyoshi, T., Furuya, T., \& Koda, M. (2010). Posterior decompression with instrumented fusion for thoracic myelopathy caused by ossification of the posterior longitudinal ligament. European Spine Journal, 19(5), 691-698.

7. Cappabianca, P., Cavallo, L. M., Esposito, I., Barakat, M., \& Esposito, F. (2010). Bone removal with a new ultrasonic bone curette during endoscopic endonasal approach to the sellar-suprasellar area. Operative Neurosurgery, 66(suppl_1), ons-E118.

8. Pavlíková, G., Foltán, R., Horká, M., Hanzelka, T., Borunská, H., \& Šedý, J. (2011). Piezosurgery in oral and maxillofacial surgery. International journal of oral and maxillofacial surgery, 40(5), 451-457.

9. Jiang, Y. Q., Li, X. L., Zhou, X. G., Bian, C., Wang, H. M., Huang, J. M., \& Dong, J. (2017). A prospective randomized trial comparing anterior cervical discectomy and fusion versus plate-only open-door laminoplasty for the treatment of spinal stenosis in degenerative diseases. European Spine Journal, 26(4), 1162-1172.

10. Li, K., Zhang, W., Li, B., Xu, H., Li, Z., Luo, D., ... \& Ma, J. (2016). Safety and efficacy of cervical laminoplasty using a piezosurgery device compared with a high-speed drill. Medicine, 95(37).

11. Yonenobu, K., Abumi, K., Nagata, K., Taketomi, E., \& Ueyama, K. (2001). Interobserver and intraobserver reliability of the Japanese Orthopaedic Association scoring system for evaluation of cervical compression myelopathy. Spine, 26(17), 1890-1894.

12. O'Brien, M. F., Peterson, D., Casey, A. T., \& Crockard, H. A. (1996). A novel technique for laminoplasty augmentation of spinal canal area using titanium miniplate stabilization: a computerized morphometric analysis. Spine, 21(4), 474-483.

13. Nakase, H., Matsuda, R., Shin, Y., Park, Y. S., \& Sakaki, T. (2006). The use of ultrasonic bone curettes in spinal surgery. Acta Neurochirurgica, 148(2), 207-213.

14. Iacoangeli, M., Neri, P., Balercia, P., Lupi, E., Di Rienzo, A., Nocchi, N., ... \& Scerrati, M. (2013). Piezosurgery for osteotomies in orbital surgery: Our experience and review of the literature. International journal of surgery case reports, 4(2), 188-191.

15. Matsuoka, H., Itoh, Y., Numazawa, S., Tomii, M., Watanabe, K., Hirano, Y., \& Nakagawa, H. (2012). Recapping hemilaminoplasty for spinal surgical disorders 
using ultrasonic bone curette. Surgical neurology international, 3.

16. Parker, S. L., Kretzer, R. M., Recinos, P. F., Molina, C. A., Wolinsky, J. P., Jallo, G. I., \& Recinos, V. R. (2013). Ultrasonic BoneScalpel for osteoplasticlaminoplasty in the resection of intradural spinal pathology: case series and technical note. Operative Neurosurgery, 73(suppl_1), ons61-ons66.

17. Hu, X., Ohnmeiss, D. D., \& Lieberman, I. H. (2013). Use of an ultrasonic osteotome device in spine surgery: experience from the first 128 patients. European Spine Journal, 22(12), 2845-2849.

18. Sanborn, M. R., Balzer, J., Gerszten, P.C., Karausky, P., Cheng, B. C., \& Welch, W. C. (2011). Safety and efficacy of a novel ultrasonic osteotome device in an ovine model. Journal of Clinical Neuroscience, 18(11), 1528-1533.

19. Bydon, M., Xu, R., Papademetriou, K., Sciubba, D. M., Wolinsky, J. P., Witham, T. F., ... \& Bydon, A. (2013). Safety of spinal decompression using an ultrasonic bone curette compared with a high-speed drill: outcomes in 337 patients. Journal of Neurosurgery: Spine, 18(6), 627-633.
20. Bydon, M., Macki, M., Xu, R., Ain, M. C., Ahn, E. S., \& Jallo, G. I. (2014). Spinal decompression in achondroplastic patients using high-speed drill versus ultrasonic bone curette: technical note and outcomes in 30 cases. Journal of Pediatric Orthopaedics, 34(8), 780-786.

21. Xing-Hua, Z. , Min, Y. , Tao, L. I. , Li, Z. , Bo, W. , \& YanBin, C. , et al. (2018). Comparison the application effect of piezosurgery and rongeur in posterior cervical expansive open-door laminoplasty. journal of clinical orthopaedics.

22. Nakase, H., Matsuda, R., Shin, Y., Park, Y. S., \& Sakaki, T. (2006). The use of ultrasonic bone curettes in spinal surgery. Acta Neurochirurgica, 148(2), 207-213.

23. Itoh, H. . (2019). Long-term results and alignment after cervical laminoplasty. The Central Japan Journal of Orthopaedic Surgery \& Traumatology.

24. Tamai, K., Suzuki, A., Yabu, A., Terai, H., Hoshino, M., Toyoda, H., ... \& Nakamura, H. (2020). Clinical Impact of Cervical Imbalance on Surgical Outcomes of Laminoplasty: A Propensity Score-Matching Analysis. Clinical Spine Surgery, 33(1), E1-E7. 